\title{
NMR Studies on Poly(oxymethylene) Crystals. II.
}

\author{
Toshiyuki ShIBATA and Shigeo Iwayanagr* \\ The Institute of Physical and Chemical Research, Wako, \\ Saitama 351, Japan.
}

(Received January 24, 1978)

\begin{abstract}
The anisotropy of NMR spectrum for poly(oxymethylene) obtained by $\gamma$ ray induced solid-state polymerization of tetraoxane was investigated as a function of the alignment angle of needle-like crystals with respect to a static magnetic field. On the basis of second moment calculations with the Gaussian-type distribution of oscillation amplitude assumed, the narrowing of the spectrum was attributed more to the rather complicated oscillations (the longitudinal oscillation along the helix axis in addition to the rotational oscillation around the helix axis of amplitude $9^{\circ}$ at $20^{\circ} \mathrm{C}$ ) than to the simple rotational oscillation around the helix axis. These complicated oscillations in poly(oxymethylene) crystal occur even at low temperatures and the amplitude of oscillation increases with increasing temperature. These results are in close agreement with those of the X-ray study of the atomic fluctuations by Chiba, et al.

KEY WORDS Poly(oxymethylene) / NMR / Molecular Motion / Anisotropy / Solid-State Polymerization / Tetraoxane /
\end{abstract}

Poly(oxymethylene) (POM) obtained from trioxane and tetraoxane single crystals by $\gamma$-ray induced solid-state polymerization ${ }^{1}$ is known to be composed of highly oriented and extended $9_{5}$ helix molecules with few chain folds and to be almost completely crystalline., ${ }^{2,3}$ POM obtained from the needle-like crystal of trioxane or tetraoxane is also a needle-like crystal, the long axis of which coincides with the helix axis of molecule. ${ }^{1}$ The anisotropy of the broad-line NMR for an aligned sample of POM obtained from trioxane was reported in a previous paper. ${ }^{4}$ However that POM crystal contained a subcrystal in the amount of about $20 \%$, and its $c$-axis (the helix axis) inclined at an angle of $76^{\circ} 7^{\prime}$ to the main crystal. ${ }^{1,6}$ Chatani, et al., ${ }^{3}$ reported that POM obtained from tetraoxane by solid-state polymerization at above $100^{\circ} \mathrm{C}$ contained no subcrystal. Therefore, the needle-like crystal polymerized from tetraoxane is well suited for studying NMR anisotropy and molecular motion within the crystal.

The molecular motion in POM crystal was investigated by the authors ${ }^{4}$ and by McBrierty, et $a l^{5} \quad$ Whereas McBrierty, et al., reported that the

* Present Address: Faculty of Technology, Gunma University, Kiryu, Gunma 376, Japan. rotational oscillation around the helix axis with the amplitude of $42^{\circ}$ at $20^{\circ} \mathrm{C}$ took place in the crystalline chain, the authors found the upper limit of the oscillation amplitude to be $30^{\circ}$ and that the molecular motion is not restricted simply to rotational oscillation around the helix axis. In this study, the anisotropy of the NMR absorption spectrum of POM polymerized from tetraoxane is investigated and molecular motion in the crystal is discussed.

\section{EXPERIMENTAL}

POM specimens used in this study are as follows. 1. Delrin $150 \mathrm{X}$ annealed for $9 \mathrm{hr}$ at $154^{\circ} \mathrm{C}$ in vacuo. The mobile fraction $F_{\mathrm{m}}$ was $27 \%$ at $20^{\circ} \mathrm{C}$.

2. The needle-like crystal obtained from trioxane by solid-state polymerization. $F_{\mathrm{m}}$ was $2 \%$ at $20^{\circ} \mathrm{C}$.

3. The needle-like crystal obtained from tetraoxane by solid-state polymerization. $F_{\mathrm{m}}$ was less than $1 \%$ at $20^{\circ} \mathrm{C}$.

The condition of the polymerization for trioxane was described in a preceding paper. ${ }^{4}$ The polymerization of tetraoxane was performed at $103^{\circ} \mathrm{C}$ by $\gamma$-ray irradiation from ${ }^{60} \mathrm{Co}$ (total dose, $2.5 \times 10^{5}$ r). Residual monomers in the polymer crystals 
were sublimated at $65^{\circ} \mathrm{C}$ under vacuum for a day and then crystals were allowed to stand for a month at room temperature. The obtained POM was a needle-like crystal about $0.5 \mathrm{~mm}$ in diameter and $0.5-1.5 \mathrm{~cm}$ in length. The rotation X-ray diagram of the crystal rotated about the long axis showed that the helical axis of the molecules coincides with the long axis of the crystal and no diffraction from the subcrystal was found. The sample used for measurements of NMR anisotropy was obtained by aligning about one hundred needle-like crystals in a Teflon sample holder.

The NMR absorption spectra were observed by a broad-line NMR apparatus ${ }^{4}$ with a PKW-type detector at $25 \mathrm{MHz}$ and a Robinson-type detector at $17 \mathrm{MHz}$. The radio frequency magnetic field $H_{1}$ was kept at the lowest amplitude possible to avoid saturation.

\section{RESULTS}

The second moments of NMR absorption spectra from -160 to $160^{\circ} \mathrm{C}$ are shown in Figure 1 for the POM sample of randomly packed needlelike crystals obtained from trioxane. In the figure, the broad components of the spectra above $-40^{\circ} \mathrm{C}$ are shown for the Delrin 150X pellet. The narrow component appears at $20^{\circ} \mathrm{C}$ for the sample obtained from trioxane and at $-40^{\circ} \mathrm{C}$ for Delrin. Above these temperatures the narrow component is ne-

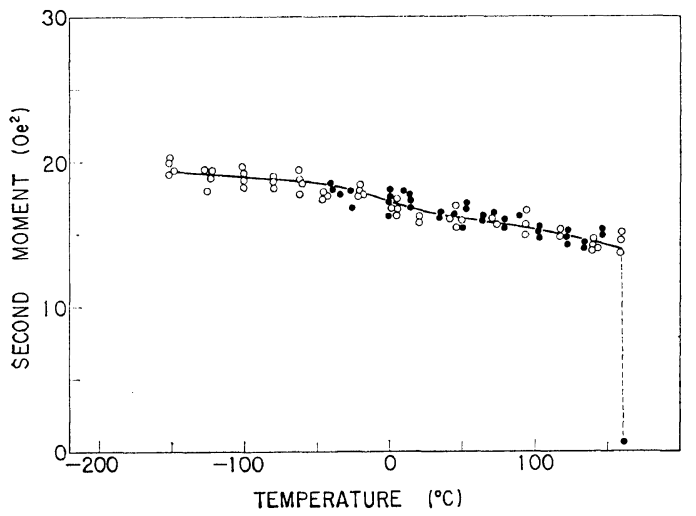

Figure 1. The second moment (broad component) for poly(oxymethylene) samples as a function of temperature: randomly packed crystals obtained from trioxane by solid-state polymerization (open circles) and Delrin 150X (filled circles); the resonance frequency at $25 \mathrm{MHz}$. glected and only the broad component of spectrum is used for calculating the second moment. The second moments for the two samples coincide with each other and narrow gradually with increasing temperature. Since the morphology and the mobile fractions of two samples are very different, the coincidence of the second moments indicates that the gradual narrowing is contributed from the crystalline region. The decrease of the second moment of POM by the thermal lattice expansion has been calculated to be $0.5 \mathrm{Oe}^{2}$ between $-197^{\circ} \mathrm{C}$ and $20^{\circ} \mathrm{C} .^{7}$ Therefore, the gradual narrowing is due to molecular motions in the crystal.

The anisotropy of NMR spectrum with respect to the alignment angle $\omega$ between the perferred axis of orientation and a static magnetic field $H_{0}$ was examined for an aligned sample obtained from tetraoxane. The line shape at $-159^{\circ} \mathrm{C}$ varies with the alignment angle $\omega$ as shown in Figure 2. The line shapes (the absorption curves) in this figure were obtained by integrating the observed derivative curves. Since the dipole-dipole interaction in a proton pair of methylene is dominant in POM (the methylene protons contribute $60 \%$ of the total second moment), it is considered that the line shape of POM is characterized largely by a twospin system. Figure 2 shows the theoretical line

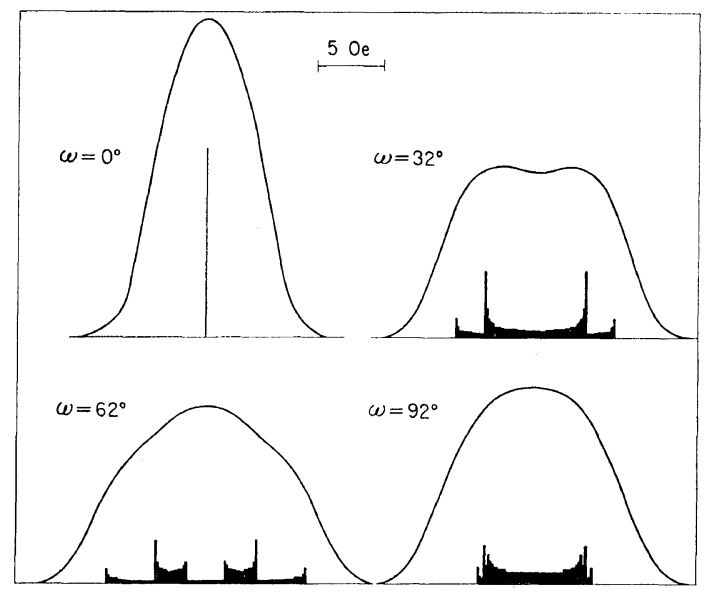

Figure 2. Observed line shapes (the absorption curves) at $-159^{\circ} \mathrm{C}$ for an aligned sample of POM obtained from tetraoxane by solid-state polymerization and theoretical line shapes for the two-spin system in perfect uniaxial orientation; the resonance frequency at $17 \mathrm{MHz}$. 
NMR Studies on Poly(oxymethylene), Crystals. II.

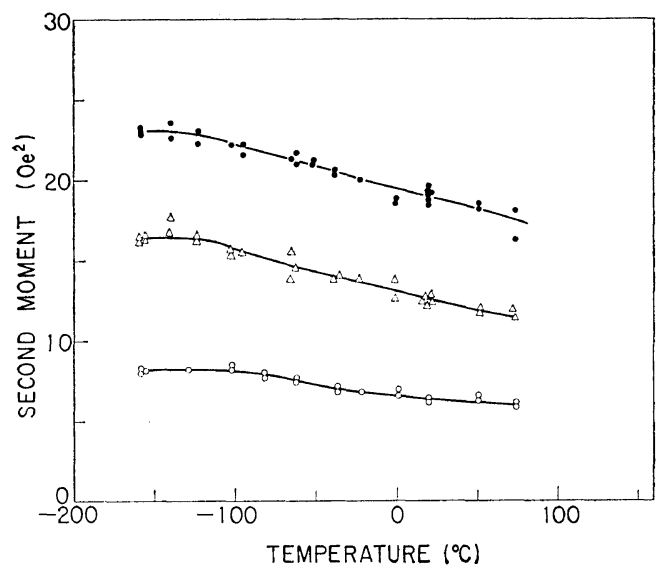

Figure 3. The second moment for an aligned sample of POM obtained from tetraoxane at $\omega=0$ (open circles), 47 (filled circles) and $92^{\circ}$ (triangles); the resonance frequency at $17 \mathrm{MHz}$.

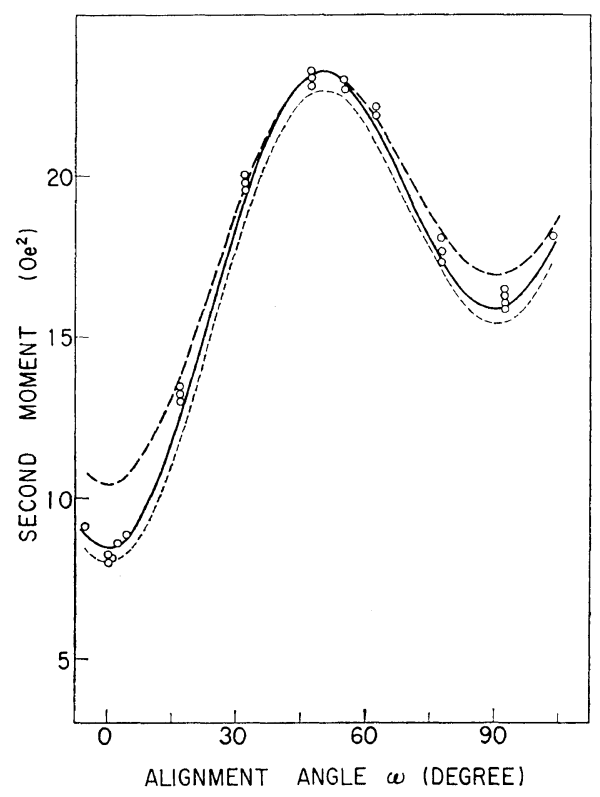

Figure 4. The observed second moment at $-159^{\circ} \mathrm{C}$ (open circles) and the theoretical ones in the rigid state as a function of the alignment angle: - - with the atomic parameters by Uchida, et al., the hexagonal lattice constant $a=4.410 \AA$ at liquid nitrogen temperature and $\delta=12^{\circ} ;$ - with the modified atomic parameters, $a=4.410 \AA$ and $\delta=9^{\circ} ;$---.--, with the modified parameters, $a=4.471 \AA$ at room temperature and $\delta=$ $9^{\circ}$.

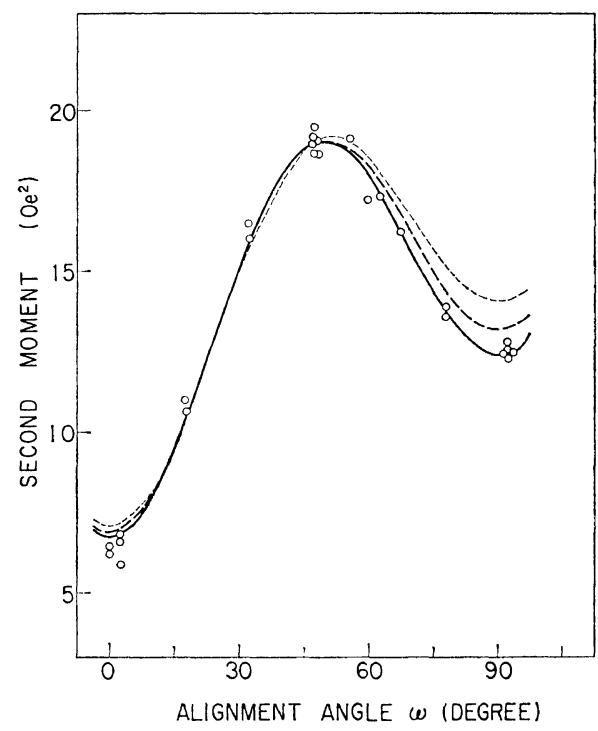

Figure 5. The observed second moment at $20^{\circ} \mathrm{C}$ (open circles) and the theoretical ones in rotational oscillations as a function of the alignment angle $\omega$ : $---, \delta_{\phi}=16^{\circ}, \delta_{\gamma}=0^{\circ} ;-\longrightarrow, \delta_{\phi}=13^{\circ}, \delta_{\gamma}=\delta_{\phi} ;-$ $\delta_{\phi}=9^{\circ}, \delta_{\gamma}=2 \delta_{\phi}$; with $\delta=9^{\circ}$.

shapes of the two-spin system in the perfect uniaxial orientation. The observed line shapes at various alignment angles agree qualitatively with the theoretical ones. A detailed study of the line shape will be reported in a later paper.

In Figure 3 is shown the second moment of the aligned sample as a function of the temperature from -159 to $80^{\circ} \mathrm{C}$ at $\omega=0^{\circ}, 47^{\circ}$, and $92^{\circ}$. The second moments at $\omega=47^{\circ}$ and $92^{\circ}$ decrease almost linearly above $-120^{\circ} \mathrm{C}$ and $\omega=0^{\circ}$ above $-100^{\circ} \mathrm{C}$. Observed second moment at $-159^{\circ} \mathrm{C}$ as a function of the alignment angle is shown in Figure 4 and at $20^{\circ} \mathrm{C}$ in Figure 5. The data shown in Figure 4 and Figure 5 represent a remarkable maximum at $\omega=45^{\circ}$ and deep minima at $\omega=0^{\circ}$ and $90^{\circ}$, the difference between them is large in comparison with that in preceding studies. ${ }^{4,5,8}$ In addition, the decrease in second moment between $-159^{\circ} \mathrm{C}$ and $20^{\circ} \mathrm{C}$ is less drastic than those reported by Olf and Peterlin ${ }^{5,8}$ on drawn specimens. This indicates that deviation from the perfectness of the crystal structure is less for the specimen used in this study than for drawn specimens used by these workers. 


\section{T. Shibata and S. Iwayanagi}

\section{DISCUSSION}

The second moment is expressed by the van Vleck equation ${ }^{9}$ :

$$
\left\langle\Delta H^{2}\right\rangle=K \sum_{i \neq j}\left[\left(3 \cos ^{2} \theta_{i j}-1\right) / r_{i j}^{3}\right]^{2}
$$

where $\theta_{i j}$ is the angle between the vector $r_{i j}$ connecting protons $i$ and $j$ and the static fleld $H_{0}$ (Figure 6), and $K$ is a constant given by $K=(3 / 4) \hbar^{2}$ $\times \gamma^{2} I(I+1) N^{-1}$.

If the rate of the motion of molecules is greater than the frequency of $\gamma \Delta H_{\text {rigid }}$, the second moment is given by the time average of the factor $\left(3 \cos ^{2} \theta_{i j}\right.$ $-1) / r_{i j}^{3}{ }^{10-12}$ :

$$
\left\langle\Delta H^{2}\right\rangle=K \sum_{i \neq j}\left\langle\left(3 \cos ^{2} \theta_{i j}-1\right) / r_{i j}^{3}\right\rangle_{t}^{2}
$$

The expression appropriate to the uniaxially oriented sample (orientation is not perfect) is derived from eq 1 or 2 as shown in Appendix:

$$
\left\langle\Delta H^{2}\right\rangle=K\left(A \cos ^{4} \omega-B \cos ^{2} \omega+C\right)
$$

In this expression $A, B$, and $C$ are independent of $\omega$ and are the functions of so called "lattice sums" and of $C_{2}$ and $C_{4}$ which represent the distribution of the molecular axis with respect to the preferred axis of orientation. Equation 3 was worked out by Olf and Peterlin. ${ }^{15}$ The general expressions (lattice sums) for the motion of the uniaxially oriented sample are given in Appendix, and are somewhat different from those given by Olf and Peterlin owing to simple errors in calculation.

In this study the distribution function $Q(\alpha)$ in eq $A-4$ is the distribution of the orientation of the long axes of needle-like crystals aligned in the Teflon sample holder. The distribution function is assumed to be gaussian with an average distribution width $\delta$ :

$$
Q(\alpha)=\frac{1}{\sqrt{2 \pi \delta}} \exp \left(-\alpha^{2} / 2 \delta^{2}\right)
$$

The crystal structure of POM has been given by Uchida, et al. ${ }^{13}$ The atomic parameters of two methylene protons have been given by assuming a bond length $\mathrm{C}-\mathrm{H}=1.09 \AA$ and the bond angle $\angle \mathrm{HCH}^{\prime}=109^{\circ} 28^{\prime}$ in the cylindrical coordinates, of which the $z$ axis coincides with the helix axis and $\phi(\mathrm{C})=z(\mathrm{C})=0$ at the position of carbon atom: $\mathrm{H}\left(1.507 \AA,-28^{\circ} 48^{\prime}, 0.515 \AA\right)$ and $\mathrm{H}^{\prime}(1.507 \AA$, $\left.28^{\circ} 48^{\prime},-0.515 \AA\right)$. $\quad H$ and $H^{\prime}$ stand for the two methylene protons. Using their parameters, the angle between the molecular axis and the methylene proton pair vector $\gamma_{\mathrm{HH}^{\prime}}$ is found to be $54^{\circ} 40^{\prime}$. The theoretical second moment in the rigid state was calculated as shown in Figure 4 with $\delta=12^{\circ}$ (the broken line) using the hexagonal lattice constant $a=4.410 \AA$ at $-196^{\circ} \mathrm{C}$ obtained by Aoki, et al. $^{7,14}$ The value of $\delta$ was determined so as to give the best fit to the observed value at $\omega=45^{\circ}$. However, the theoretical curve with $\delta=12^{\circ}$ deviates from the observed one at about $\omega=0$ and $90^{\circ}$. In order to have the best fit over the entire range of $\omega$, a small modification of the atomic parameters of Uchida, et al., is needed, maintaining the relations $r_{\mathrm{H}}=r_{\mathrm{H}^{\prime}}, \phi_{\mathrm{H}}=-\phi_{\mathrm{H}^{\prime}}$, and $z_{\mathrm{H}}=-z_{\mathrm{H}^{\prime}}$ and the bond length $\mathbf{C}-\mathbf{H}=1.09 \AA$. The modified parameters are $\mathrm{H}\left(1.505 \AA,-30^{\circ} 4^{\prime}, 0.489 \AA\right)$ and $\mathrm{H}^{\prime}(1.505 \AA$, $30^{\circ} 4^{\prime},-0.489 \AA$ ). The $\gamma_{\mathrm{HH}^{\prime}}$ is $57^{\circ}$ and $\angle \mathrm{HCH}^{\prime}=$ $110^{\circ} 48^{\prime}$ with these parameters. The theoretical curve with $\delta=9^{\circ}$ calculated by our parameters is shown as the solid line in Figure 4. The value of $\delta$ is determined again so as to give the best fit to the observed value at $\omega=45^{\circ}$. In what follows, the modified parameters and $\delta=9^{\circ}$ are used for the second moment calculations.

The decrease in the second moment by the thermal lattice expansion (the difference between the solid line and the dotted line with $a=4.471 \AA$ at room temperature ${ }^{13}$ in Figure 4 ) is about $0.5 \mathrm{Oe}^{2}$ over the entire range of the alignment angles. Therefore, the difference between the dotted line in Figure 4 and observed values at $20^{\circ} \mathrm{C}$ in Figure 5 is attributed to molecular motions in the crystalline chains. The methylene proton pair is assumed to undergo the Gaussian oscillation ${ }^{15}$ of the angle $\phi_{\mathrm{HH}^{\prime}}$ and $\gamma_{\mathrm{HH}^{\prime}}$, where the probability of finding the proton pair at a displacement angle of $\Delta \phi$ or $\Delta \gamma$ is given by normal distributions of width $\delta_{\phi}$ or $\delta_{r}$ :

$$
\begin{aligned}
& p(\Delta \phi)=\frac{1}{\sqrt{2 \pi} \delta_{\phi}} \exp \left(-\Delta \phi^{2} / 2 \delta_{\phi}{ }^{2}\right) \\
& p(\Delta \gamma)=\frac{1}{\sqrt{2 \pi} \delta_{\gamma}} \exp \left(-\Delta \gamma^{2} / 2 \delta_{\gamma}^{2}\right)
\end{aligned}
$$

Also, the oscillations of $\phi_{\mathrm{HH}^{\prime}}$ and $\gamma_{\mathrm{HH}^{\prime}}$ for methylene are assumed to be independent of each other. The equations for the methylene gaussian oscillations are expressed by (A-8) in Appendix. Olf and Peterlin ${ }^{15}$ have shown that the other type 
of rotational oscillation (harmonic or statistical oscillation) gives results very similar to those from the gaussian oscillation.

Interactions between neighbouring chains are calculated numerically assuming independent oscillations using eq A-5-A-7 in Appendix. However, the oscillations of methylenes in a chain are assumed to be coherent, since the second moment between methylenes in the chain increased with an increase in the oscillation width, if $\delta_{\phi}$ or $\delta_{r}$ is beyond $5^{\circ}$.

In Figure 5 are shown the theoretical second moments for the gaussian oscillations of (i) $\delta_{\phi}=16^{\circ}$ and $\delta_{\gamma}=0^{\circ}$ (the dotted line), (ii) $\delta_{\phi}=13^{\circ}$ and $\delta_{\gamma}=$ $\delta_{\phi}$ (the broken line) and (iii) $\delta_{\phi}=9^{\circ}$ and $\delta_{\gamma}=2 \delta_{\phi}$ (the solid line). The oscillation widths were so determined that the theoretical value is fitted to the observed value at $\omega=45^{\circ}$. The curve at $\delta_{r}=0^{\circ}$ corresponds that case in which the molecular motion is restricted to the rotational oscillation around the helix axis. The observed values at $20^{\circ} \mathrm{C}$ fall closely on the curve of $\delta_{\gamma}=2 \delta_{\phi}$. This fact suggests that POM molecules in the crystal undergo complicated oscillational motions of the oscillation widths of $\delta_{\phi}=9^{\circ}$ and $\delta_{\gamma}=18^{\circ}$ rather than the simple rotational oscillation around the helix axis. Figure 6 shows the oscillation width $\delta_{\phi}$ estimated by this procedure at various temperatures. The amplitude of oscillation increases with increasing temperature.

By measuring the temperature-dependence of the intensities of X-ray reflections, Chiba, et al., ${ }^{16}$

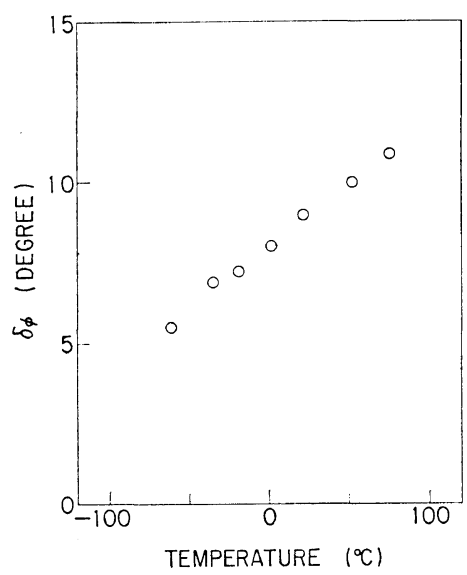

Figure 6. Temperature dependence of the oscillation width $\delta_{\phi}$. found atomic fluctuations with large amplitudes in POM crystal above $-100^{\circ} \mathrm{C}$. They estimated the root mean square average of the atomic fluctuations to be $\left\langle\Delta \phi^{2}\right\rangle^{1 / 2}=12^{\circ}$ and $\left\langle\Delta z^{2}\right\rangle^{1 / 2}=0.1 \AA$ at room temperature. The atomic fluctuations in the $z$ direction studied by the X-ray method were considered mainly to be translational motions. Hence the motions in the $z$ direction by NMR and $\mathrm{X}$-ray method cannot be compared directly with each other. Nevertheless the amplitude of oscillation around the helix axis in the present NMR study agrees well with that by Chiba $e t$ al.

\section{APPENDIX}

\section{General Equations}

$\cos \theta_{i j}$ in eq 1 or 2 is replaced by the following equation:

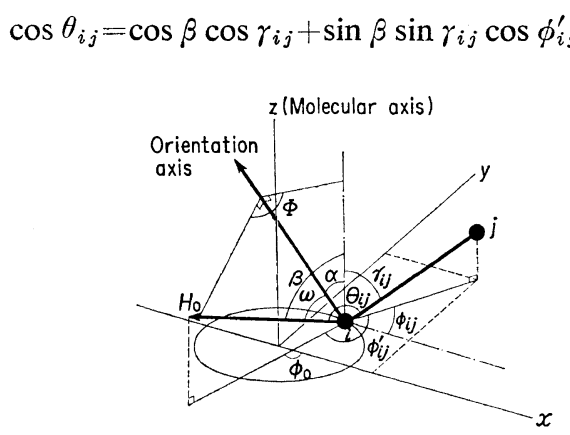

(A-1)

Figure 7. Definition of angles.

where the angles are defined in Figure 7. After $\phi^{\prime}$ is replaced by $\left(\phi_{0}+\phi\right)$, the equation is averaged with respect to $\phi_{0}$. Then $\cos \beta$ is eliminated by the equation:

$\cos \beta=\cos \alpha \cos \omega+\sin \alpha \sin \omega \cos \Phi$

By averaging the equation with respect to $\Phi$ and $\alpha$, the expression appropriate to the uniaxially oriented sample (orientation is not perfect) is obtained:

$$
\left\langle\Delta H^{2}\right\rangle=K\left(A \cos ^{4} \omega-B \cos ^{2} \omega+C\right)
$$

In this expression $A, B$, and $C$ are defined by the following equations:

$A=(1 / 8) D\left(35 C_{4}-30 C_{2}+3\right)$

$B=(3 / 4) D\left(5 C_{4}-6 C_{2}+1\right)+(1 / 2) E\left(1-3 C_{2}\right)$

$C=(3 / 8) D\left(1-2 C_{2}+C_{4}\right)+(1 / 2) E\left(1-C_{2}\right)+F(\mathrm{~A}-3)$ 


\section{T. Shibata and S. Iwayanagi}

The distribution of the molecular axis with respect to the preferred axis of orientation is expressed by $C_{2}$ and $C_{4}$ :

$$
\begin{aligned}
& C_{2}=\left\langle\cos ^{2} \alpha\right\rangle=\int_{0}^{\pi / 2} Q(\alpha) \cos ^{2} \alpha \sin \alpha \mathrm{d} \alpha \\
& C_{4}=\left\langle\cos ^{4} \alpha\right\rangle=\int_{0}^{\pi / 2} Q(\alpha) \cos ^{4} \alpha \sin \alpha \mathrm{d} \alpha
\end{aligned}
$$

where $Q(\alpha)$ is the distribution function normalized to unity.

The lattice sums $D, E$, and $F$ in eq A-3 are:

$$
\begin{aligned}
& D=9 G_{1}{ }^{2}-M+N-P \\
& E=M-2 N+P+Q-6 G_{1} G_{7} \\
& F=N-Q+G_{7}{ }^{2}
\end{aligned}
$$

where $M, N, P$, and $Q$ are expressed by the following equations:

$$
\begin{aligned}
& M=(9 / 2)\left(G_{4}{ }^{2}+G_{6}{ }^{2}\right) \\
& N=(9 / 8)\left(3 G_{2}{ }^{2}+G_{5}{ }^{2}+3 G_{3}{ }^{2}+2 G_{2} G_{3}\right) \\
& P=9\left(G_{1} G_{2}+G_{1} G_{3}\right) \\
& Q=3\left(G_{2} G_{7}+G_{3} G_{7}\right)
\end{aligned}
$$

In these expressions $G_{1}-G_{7}$ are the time averages of the functions of $\gamma_{i j}, \phi_{i j}$, and $r_{i j}$ :

$$
\begin{aligned}
& G_{1}=\left\langle\cos ^{2} \gamma_{i j} / r_{i j}^{3}\right\rangle_{t} \\
& G_{2}=\left\langle\sin ^{2} \gamma_{i j} \cos ^{2} \phi_{i j} \mid r_{i j}^{3}\right\rangle_{t} \\
& G_{3}=\left\langle\sin ^{2} \gamma_{i j} \sin ^{2} \phi_{i j} \mid r_{i j}^{3}\right\rangle_{t} \\
& G_{4}=\left\langle\sin 2 \gamma_{i j} \cos \phi_{i j} \mid r_{i j}^{3}\right\rangle_{t} \\
& G_{5}=\left\langle\sin ^{2} \gamma_{i j} \cos 2 \phi_{i j} / r_{i j}^{3}\right\rangle_{t} \\
& G_{6}=\left\langle\sin 2 \gamma_{i j} \sin \phi_{i j} / r_{i j}^{3}\right\rangle_{t} \\
& G_{7}=\left\langle 1 / r_{i j}^{3}\right\rangle_{t}
\end{aligned}
$$

The equation 3 combined by eq A-3-A-7 gives the equations by $\mathrm{Olf}^{22}$ if $C_{2}=C_{4}=1$ is assumed.

\section{Gaussian Oscillation of Methylene}

If we consider the proton pair of methylene undergoing the gaussian oscillations, $r_{i j}$ is constant and $\phi_{i j}$ and $\gamma_{i j}$ are displaced by $\Delta \phi$ and $\Delta \gamma$ from respective equilibrium values $\phi_{i j}^{0}$ and $\gamma_{i j}^{0}$ :

$$
\begin{aligned}
& \phi_{i j}=\phi_{i j}^{0}+\Delta \phi \\
& \gamma_{i j}=\gamma_{i j}^{0}+\Delta \gamma
\end{aligned}
$$

where $\Delta \phi_{i j}$ and $\Delta \gamma_{i j}$ are assumed to distribute according to eq 5 and 6 . These are substituted in eq A-7 and using the time averages summarized in Table I of ref 15 , we obtain the expression for methylene undergoing the gaussian oscillations of $\phi_{i j}$ and $\gamma_{i j}$ independently after averaging with respect to $\phi_{i j}^{0}$ :

$$
\begin{aligned}
& M=(9 / 2) b^{2} \exp \left(-\delta_{\phi}^{2}\right) / r^{6} \\
& N=(9 / 32)(1-a)^{2}\left[2+\exp \left(-4{\delta_{\phi}}^{2}\right)\right] / r^{6} \\
& P=(9 / 4)\left(1-a^{2}\right) / r^{6} \\
& Q=(3 / 2)(1-a) / r^{6} \\
& G_{1}{ }^{2}=(1 / 4)(1+a)^{2} / r^{6} \\
& G_{1} G_{7}=(1 / 2)(1+a) / r^{6} \\
& G_{7}{ }^{2}=1 / r^{6}
\end{aligned}
$$

where $a$ and $b$ are defined as follows:

$$
\begin{aligned}
& a=\cos 2 \gamma_{i j}^{0} \exp \left(-2 \delta_{r}{ }^{2}\right) \\
& b=\sin 2 \gamma_{i j}^{0} \exp \left(-2 \delta_{\gamma}{ }^{2}\right)
\end{aligned}
$$

If $\delta_{\phi}=\delta_{r}=0^{\circ}$, these expressions coincide with those in the rigid state. ${ }^{8,23}$

Acknowledgments. The authors are grateful to Professors A. Chiba, K. Hikichi, and M. Kaneko for their valuable comments and to Mr. J. Uzawa for his assistance in maintaining the NMR apparatus.

\section{REFERENCES}

1. K. Hayashi, M. Nishi, and S. Okamura, J. Polym. Sci., Part C, 4, 839 (1963).

2. A. I. Slutsker, A. E. Gromov, and V. S. Pshezhetskii, Soviet Phys.-Solid-State, 6, 362 (1964).

3. Y. Chatani, T. Uchida, and H. Tadokoro, $J$. Macromol. Sci-Phys., B2, 567 (1968).

4. S. Iwayanagi and I. Sakurai, J. Macromol. Sci.Phys., B3, 259 (1969).

5. V. J. McBrierty, and I. R. McDonald, J. Phys. Appl. Phys., 6, 131 (1973).

6. G. Carazzolo, S. Leghissa, and M. Mammi, Makromol. Chem., 60, 171 (1963).

7. K. Hikichi, A. Tsutsumi, J. Oda, and M. Kaneko, Polym. J., 6, 56 (1974).

8. H. G. Olf, and A. Peterlin, J. Appl. Phys., 35, 3108 (1964).

9. J. H. Van Vleck, Phys. Rev., 74, 1168 (1948).

10. H. S. Gutowsky, and G. E. Pake, J. Chem. Phys., 
NMR Studies on Poly(oxymethylene), Crystals. II.

18, 162 (1950).

11. E. R. Andrew, J. Chem. Phys., 18, 607 (1950).

12. E. R. Andrew, and R. A. Newing, Proc. Phys. Soc. London, 72, 959 (1958).

13. T. Uchida, and H. Tadokoro, J. Polym. Sci.,Part $A-2,5,63$ (1967).

14. Y. Aoki, A. Chiba, and M. Kaneko, J. Phys. Soc. Jpn., 27, 1579 (1969).

15. H. G. Olf, and A. Peterlin, J. Polym. Sci., Part $A-2,8,753$ (1970).

16. A. Chiba, Y. Aoki, K. Hikichi and M. Kaneko, Rep. Prog. Polym. Phys. Jpn., 11, 213 (1968).

17. A. Chiba, A. Hasegawa, K. Hikichi, and J. Furui- chi, J. Phys. Soc. Jpn., 21, 1777 (1966).

18. H. G. Olf, and A. Peterlin, J. Polym. Sci., Part A-2, 8, 771 (1970).

19. D. Hyndman, and G. F. Origlio, J. Appl. Phys., 31, 1849 (1960).

20. I. Sakurada, T. Ito, and K. Nakamae, J. Polym. Sci., Part C, 15, 75 (1966).

21. I. Sakurada, and K. Kaji, J. Polym. Sci. Part C. 31, 57 (1970).

22. H. G. Olf, J. Polym. Sci., Part A-2, 9, 1851 (1971).

23. K. Yamagata, and S. Hirota, Rep. Prog. Polym. Phys., Jpn., 5, 236 (1962). 